\title{
Association Between Serological Markers and Crohn's Disease Activity
}

\author{
Zunirah Ahmed ${ }^{\mathrm{a}}$, Michael Lysek ${ }^{\mathrm{b}}$, Nan Zhang ${ }^{\mathrm{c}}$, Talha A. Malik ${ }^{\mathrm{d}, \mathrm{e}}$
}

\begin{abstract}
Background: The aim was to study the association between six serological markers and Crohn's disease (CD) activity at an inflammatory bowel disease (IBD) referral center.

Methods: We designed a retrospective cohort study using adults (> 18 years) with $\mathrm{CD}$ followed for at least 1 year at University of Alabama at Birmingham. Baseline serological markers ASCA-IgA, ASCA-IgG, anti-OmpC IgA, anti-CBir1 IgG, anti-A4Fla2 IgG and anti-FlaX IgG were drawn at initial visit. Poisson regression was used to assess the longitudinal relationship between these markers drawn at baseline and rate of active clinical disease during follow-up.
\end{abstract}

Results: Each marker, from 135 patients, was categorized into high vs. low. A Poisson regression model adjusted for age, gender, race, duration of disease, obesity, proton pump inhibitor; steroid and thiopurine use, and disease location demonstrated that $\mathrm{CD}$ patients with high anti-CBir1 IgG at baseline were approximately twice more likely to have active clinical disease (incidence rate ratio (IRR) $2.06,95 \%$ confidence interval $(\mathrm{CI}) 1.28-3.33, \mathrm{P}=0.0032)$. The unadjusted Poisson regression model for A4Fla2 IgG antibody level did suggest that a high A4Fla2 IgG at baseline was associated with a higher likelihood of active CD (IRR 1.64, 95\% CI 1.07, 2.53, $\mathrm{P}=0.0238$ ) which however, upon adjustment based on effect size, was not significant. The other four antibodies did not appear to predict clinical course.

Conclusions: High levels of anti-CBir1 IgG appear to be associated with a greater likelihood of active $\mathrm{CD}$. Whether routine baseline testing for anti-CBir1 IgG to predict a more active clinical course is warranted needs more research.

Keywords: Crohn's disease; Antibodies; CBir IgG

Manuscript submitted October 25, 2019, accepted November 20, 2019

aDepartment of Medicine, University of Alabama at Birmingham Montgomery, AL 36116, USA

bDepartment of Medicine, University of South Alabama, Mobile, AL 36617, USA

'Department of Biostatistics, Mayo Clinic, Scottsdale, AZ 85259, USA

dDepartment of Medicine-Gastroenterology, Mayo Clinic, Scottsdale, AZ 85259 , USA

'Corresponding Author: Talha A. Malik, Department of Medicine-Gastroenterology, Mayo Clinic, Scottsdale, AZ 85259, USA.

Email: Malik.Talha@mayo.edu

doi: https://doi.org/10.14740/jocmr4016

\section{Introduction}

Crohn's disease (CD) is a chronic relapsing-remitting inflammation of the gastrointestinal tract. It is a prototypical complex disorder with several factors including environmental triggers, immune response to gut microbiota, genetic susceptibility and dietary factors playing a role in the pathogenesis [1]. Currently the diagnosis of $\mathrm{CD}$ requires invasive endoscopic, radiologic and histopathologic criteria [2]. In recent years, the focus of inflammatory bowel disease (IBD) research has shifted towards the development of non-invasive tests that can potentially augment or replace part of the diagnostic process.

IBD is characterized by production of several serological antibodies which are mainly divided into autoantibodies and microbial antibodies [3]. Autoantibodies are antibodies produced against intestinal and non-intestinal components, whereas microbial antibodies are in response to microorganisms including yeast, bacteria and fungi [4]. The most popular antibodies studied in relation to $\mathrm{CD}$ are nuclear lamina protein which is present in neutrophils (perinuclear anti-neutrophilic cytoplasmic antibody (pANCA)) and antibodies against mannose epitopes from the yeast Saccharomyces cerevisiae (antiSaccharomyces cerevisiae antibody (ASCA)) [5]. Currently newer antibodies like anti-OmpC and anti-L have been found to be associated with $\mathrm{CD}$ [6]. The diagnostic utility of these serological markers in differentiating IBD subtypes (CD vs. ulcerative colitis (UC)), along with predicting disease course and treatment outcomes, poses a clinical challenge for practitioners due to a lack of clinical trials.

This study aimed to evaluate the effect of different serological markers on $\mathrm{CD}$ outcome in terms of clinical disease activity.

\section{Materials and Methods}

\section{Study design, patient population and selection criteria}

We conducted a retrospective cohort study to evaluate the association between serological markers and rate of active $\mathrm{CD}$ in patients at University of Alabama at Birmingham (UAB), a tertiary care IBD referral center. The study population included adult $\mathrm{CD}$ patients seen at the UAB IBD center from 2014 to 2018. Inclusion criteria included CD patients identified based on the sampling of serum genetic inflammatory 
(SGI) marker profile from electronic medical record (EMR) baseline and then followed to assess CD activity at different IBD clinic visits. All included patients had at least two visits during a given year.

Exclusion criteria included patients with poor or incomplete EMR documentation, those who were diagnosed with colorectal or another cancer, developed any severe infection or reaction, underwent any CD-related surgery, had a CD-related hospital admission, and women who were noted to be pregnant during the period of observation.

\section{Data collection and variable definitions}

Data were collected through retrospective and prospective review of EMRs. Data collected at the time of the first observation in our tertiary referral center included age, race, gender, duration of disease, location and behavior of $\mathrm{CD}$, nicotine use, proton pump inhibitor (PPI) use, vitamin D level, bone mineral density, presence of metabolic syndrome and its components, and biologic (vedolizumab/tumor necrosis factor (TNF) blocker) experience.

Data collected from the full period of observation included time from first clinical contact to subsequent clinic visits. Data on additional CD therapy during induction (i.e. steroids, thiopurine analogue and methotrexate) were also collected.

The exposure of interest comprised CD patients with an SGI marker profile at baseline and then followed subsequently for clinical CD activity. Harvey-Bradshaw index (HBI) was used to assess the clinical disease activity. Inactive or mild disease was defined as HBI $<8$ and moderate to severe disease was defined as $\mathrm{HBI}>8$.

Nicotine use was defined as documented ongoing use at initial visit. PPI use was defined based on medication documentation in EMR at first visit. Steroid use was defined as exposure post- induction to rectal, topical, or oral corticosteroids for at least 4 weeks. Thiopurine use was defined as use of azathioprine or 6-mercaptopurine for at least 4 weeks during observation. Methotrexate use was defined as use of methotrexate for at least 4 weeks during period of observation. Montreal classification was used to define location and behavior of CD.

\section{Statistical analysis}

We conducted descriptive analysis for covariates by exposure groups (antibody high level vs. antibody low level). $T$-test or Wilcoxon rank sum test was used to compare continuous variables and Chi-square test or Fisher's exact test was used to compare categorical variables when applicable. Unadjusted and adjusted Poisson regression models were used to estimate rate ratios (RRs) and 95\% confidence intervals (95\% CIs) for active clinical disease. Potential confounders for inclusion into adjusted Poisson regression models were selected based on their effect size (percent change of adjusted odds ratio (OR) from unadjusted OR) of $15 \%$ or more. All statistical analyses were conducted using SAS 9.4. The current study was approved by UAB's Office of Institutional Review Board.
Table 1. Baseline Characteristics of Patients

\begin{tabular}{|c|c|}
\hline & $\begin{array}{l}\text { Crohn's patients } \\
\text { with SGI at baseline }\end{array}$ \\
\hline Age, mean (SD) & $43.9(15.7)$ \\
\hline \multicolumn{2}{|l|}{ Sex, N (\%) } \\
\hline Females & $85(63 \%)$ \\
\hline Males & $50(37 \%)$ \\
\hline \multicolumn{2}{|l|}{ Race, N (\%) } \\
\hline Caucasians & $82(60.7 \%)$ \\
\hline African Americans & $49(36.3 \%)$ \\
\hline Others & $4(3.0 \%)$ \\
\hline Duration of disease in years, mean (SD) & $9.6(11.0)$ \\
\hline Steroid use, N (\%) & $57(42.5 \%)$ \\
\hline Tobacco use, $\mathrm{N}(\%)$ & $30(22.4 \%)$ \\
\hline TNF blocker use, $\mathrm{N}(\%)$ & $87(64.9 \%)$ \\
\hline VD use, $\mathrm{N}(\%)$ & $20(14.9 \%)$ \\
\hline UST use, N (\%) & $36(26.9 \%)$ \\
\hline Thiopurine, N (\%) & $21(15.7 \%)$ \\
\hline MTX, N (\%) & $16(11.9 \%)$ \\
\hline \multicolumn{2}{|l|}{ Crohn's behavior, N (\%) } \\
\hline Penetrating & $52(38.8 \%)$ \\
\hline Stricturing & $35(26.1 \%)$ \\
\hline None & $47(35.1 \%)$ \\
\hline Perianal, N (\%) & $40(29.9 \%)$ \\
\hline UGI, N (\%) & $30(22.4 \%)$ \\
\hline \multicolumn{2}{|l|}{ Crohn's location, N (\%) } \\
\hline Ileal & $23(17 \%)$ \\
\hline Colonic & $39(28.9 \%)$ \\
\hline Ileocolonic & $72(53.3 \%)$ \\
\hline BMI, mean (SD) & $26.8(7.2)$ \\
\hline Obesity, N (\%) & $48(35.8 \%)$ \\
\hline PPI use, N (\%) & $42(31.3 \%)$ \\
\hline
\end{tabular}

SD: standard deviation; SGI: serum genetic inflammatory; TNF: tumor necrosis factor; VD: vedolizumab; UST: ustekinumab; MTX: methotrexate; UGI: upper gastrointestinal; BMI: body mass index; PPI: proton pump inhibitor.

\section{Results}

A total of 135 patients with CD who had SGI markers drawn at initial visit and subsequent clinic visits were analyzed. The six serological markers ASCA-IgA, ASCA-IgG, anti-OmpC IgA, anti-CBir1 IgG, anti-A4Fla2 IgG and anti-FlaX IgG were dichotomously divided into high and low. The baseline characteristics of the patients included in the final sample are shown in Table 1. The final sample included $85(63 \%)$ females and $53(37 \%)$ males. The mean duration of disease was 9.6 years with standard deviation (SD) of 11 . Amongst these patients, $52(38.8 \%)$ had penetrating disease and 35 (26.1\%) had stric- 
Table 2. Characteristics of Sample of Crohn's Patients by Anti-CBir1 IgG Category

\begin{tabular}{|c|c|c|c|}
\hline & Low anti-CBir1 IgG & High anti-CBir1 IgG & P value \\
\hline Age, mean (SD) & $45.4(15.8)$ & $39.7(14.8)$ & $0.0692^{\mathrm{a}}$ \\
\hline Sex, N (\%) & & & $0.2871^{\mathrm{b}}$ \\
\hline Males & $40(39.6 \%)$ & $10(29.4 \%)$ & \\
\hline Race, N (\%) & & & $0.2417^{\mathrm{c}}$ \\
\hline Others & $2(2.0 \%)$ & $2(5.9 \%)$ & \\
\hline Duration of disease in years, mean (SD) & $9.7(11.0)$ & $9.3(11.3)$ & $0.8989^{d}$ \\
\hline Steroid use, N (\%) & $38(37.6 \%)$ & $19(57.6 \%)$ & $0.0441^{\mathrm{b}}$ \\
\hline Tobacco use, N (\%) & $22(21.8 \%)$ & $8(24.2 \%)$ & $0.7685^{b}$ \\
\hline Thiopurine, N (\%) & $13(12.9 \%)$ & $8(24.2 \%)$ & $0.1188^{b}$ \\
\hline MTX, N (\%) & $13(12.9 \%)$ & $3(9.1 \%)$ & $0.5609^{\mathrm{b}}$ \\
\hline Crohn's behavior, N (\%) & & & $0.5271^{b}$ \\
\hline Penetrating & $37(36.6 \%)$ & $15(45.5 \%)$ & \\
\hline Stricturing & $26(25.7 \%)$ & $9(27.3 \%)$ & \\
\hline None & $38(37.6 \%)$ & $9(27.3 \%)$ & \\
\hline Perianal, N (\%) & $31(30.7 \%)$ & $9(27.3 \%)$ & $0.7093^{\mathrm{b}}$ \\
\hline UGI, N (\%) & $21(20.8 \%)$ & $9(27.3 \%)$ & $0.4381^{b}$ \\
\hline Crohn's location, N (\%) & & & $0.0251^{\mathrm{b}}$ \\
\hline
\end{tabular}

aTwo sample $t$-test; ${ }^{b}$ Chi-square; 'Fisher's exact; ${ }^{d}$ Wilcoxon rank sum. SD: standard deviation; TNF: tumor necrosis factor; VD: vedolizumab; UST: ustekinumab; MTX: methotrexate; UGI: upper gastrointestinal; BMI: body mass index; PPI: proton pump inhibitor.

turing disease. Perianal involvement was seen in $40(29.9 \%)$ of the patients, ileocolonic disease was most common in 72 $(53.3 \%)$ patients followed by colonic in $39(28.9 \%)$ patients and then ileal disease in $23(17 \%)$ patients.

Tables 2 and 3 highlight the characteristics of patients by anti-CBir1 IgG and the A4Fla2 IgG antibody levels.

Poisson regression model adjusted for age, gender, race, duration of disease, obesity, PPI use, steroid, thiopurine and Crohn's behavior and location demonstrated that CD patients with high anti-CBir1 IgG antibody level at baseline were approximately twice more likely to have active clinical disease during observation (IRR 2.06, 95\% CI 1.28 - 3.33, $\mathrm{P}=0.0032$ ). The unadjusted Poisson regression model for A4Fla2 IgG antibody level did suggest that a high A4Fla2 IgG antibody level at baseline was associated with a higher likelihood of active
CD (IRR 1.64, 95\% CI $1.07-2.53, \mathrm{P}=0.0238$ ); however, on adjustment based on effect size, this association did not remain statistically significant (IRR 1.55, 95\% CI $0.95-2.52, \mathrm{P}=$ 0.0789). The other four antibodies did not appear to predict a more severe clinical course. The results are further described in Table 4.

\section{Discussion}

Our study demonstrates that high anti-CBir IgG levels are associated with a more severe clinical course of CD. Anti-CBir 1 antibody is produced against the CBir flagellin found on Clostridium spp. The CBir flagellin via interaction between $\mathrm{B}$

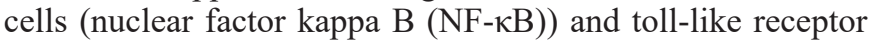


Table 3. Characteristics of Sample of Crohn's Patients by Anti-A4Fla2lgG Category

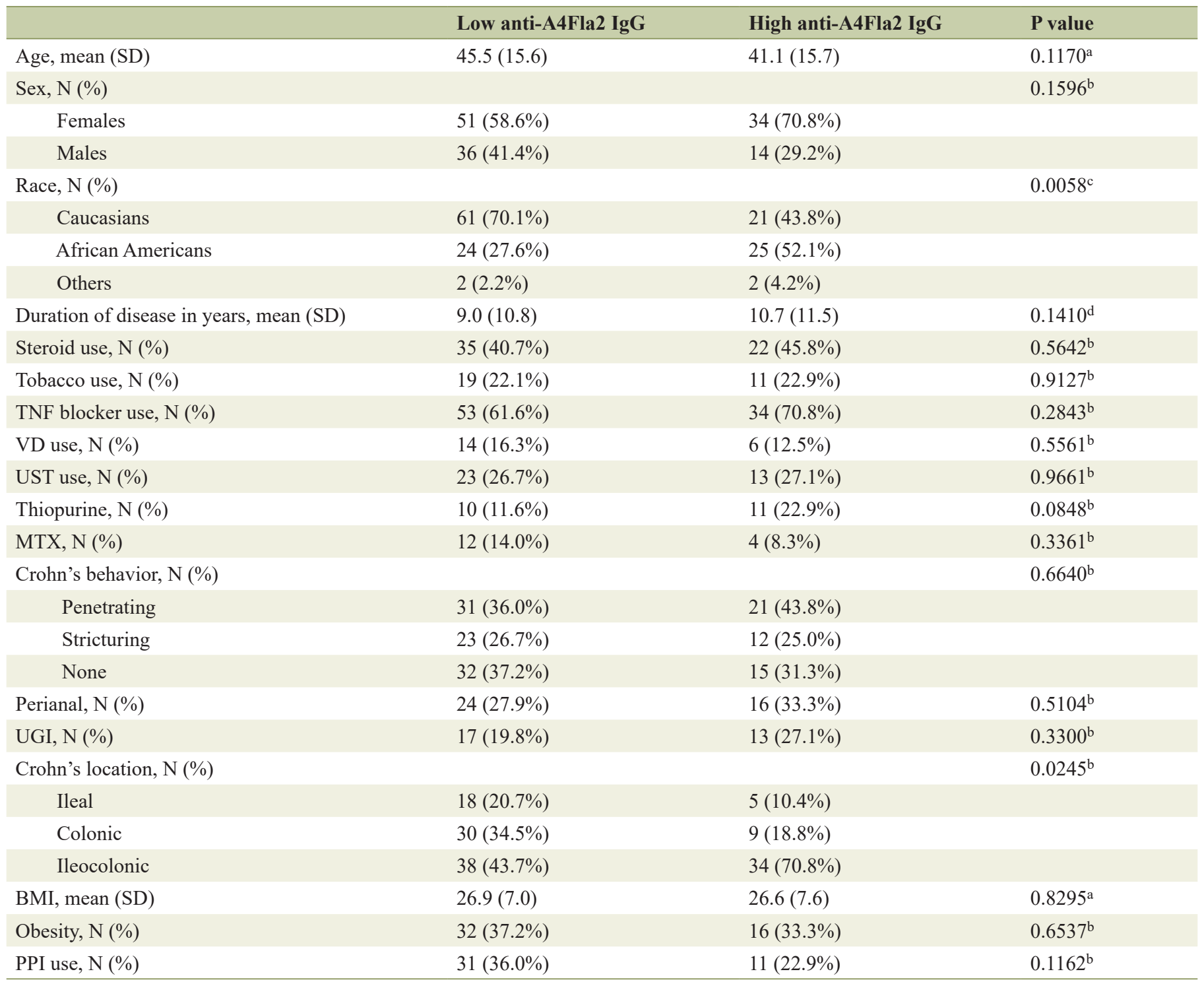

aTwo sample $t$-test; ${ }^{b}$ Chi-square; 'Fisher's exact; ${ }^{d}$ Wilcoxon rank sum. SD: standard deviation; TNF: tumor necrosis factor; VD: vedolizumab; UST: ustekinumab; MTX: methotrexate; UGI: upper gastrointestinal; BMI: body mass index; PPI: proton pump inhibitor.

5 (TLR5) induces many proinflammatory cytokines [7]. CBir antibody is commonly associated with $\mathrm{CD}$ and its expression in $\mathrm{CD}$ patients is independently associated with fibrostenosing disease and complicated small bowel CD [8, 9]. A study of UC patients demonstrated that ASCA and anti-CBir are associated with development of $\mathrm{CD}$ and chronic pouchitis in UC patients undergoing ileal pouch anal anastomosis [10]. Another study showed anti-CBir1 antibody seropositivity was significantly associated with increased health care resource utilization in CD patients as this subset of the patient population tends to have a more severe and complicated disease course [11].

Prior studies have shown that serological markers ASCAIgA, ASCA-IgG, OmpC, CBir1, ANCA and pANCA are associated with IBD. These markers are also known for their ability to discriminate between $\mathrm{CD}$ and $\mathrm{UC}[12,13]$. However, incorporating serological, genetic and inflammatory markers in the diagnostic algorithm has more accuracy of diagnosing IBD and differentiating UC and CD compared to serological markers alone [14]. Cross-sectional data analysis has further shown that the combination of serological markers and NOD genetic markers may provide physicians with a tool to assess the probability of patients who would develop complicated CD [15].

This study had several limitations. The most important limitation was the small sample size which may impact generalizability; another limitation was the observational and mostly retrospective nature of this study. Furthermore, several factors had to be adjusted because of their effect size. An important limitation was the lack of values of serological markers after baseline testing and therefore our inability to capture any 

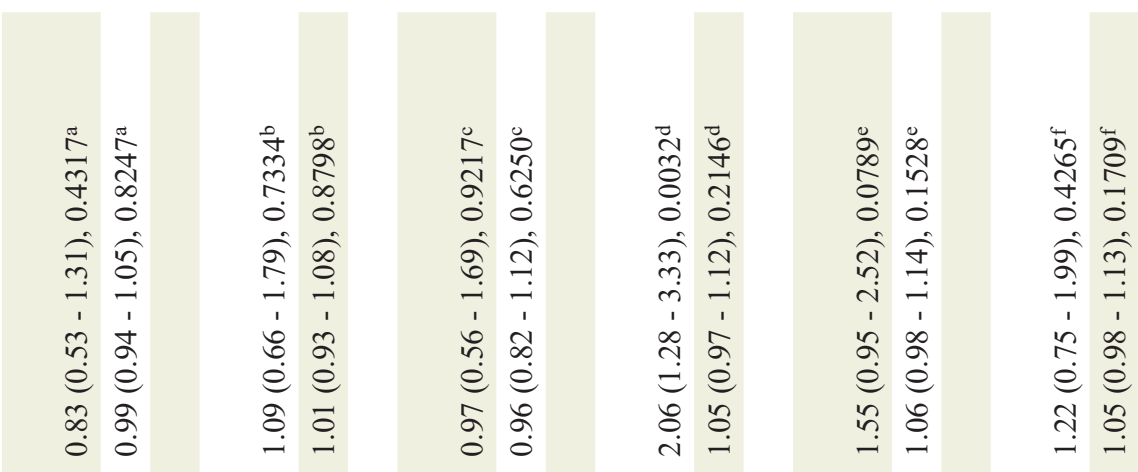

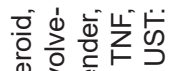

这

बi

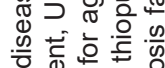

$\therefore$

$\stackrel{\sigma}{\sigma}$

-

$\stackrel{\leftrightarrow}{\stackrel{0}{e}}$

ô.

ล. ڤ̊.

i

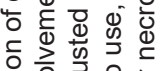

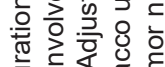

궁 $\frac{\pi}{0}$

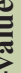

¿

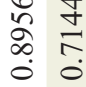

กิ้

0
0
0
0
0
0

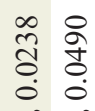

हิ)

f

क्षे

$\rightarrow-1$

mं

77

กิ

eे

ถ้

eे

웅

ô.

ㅎ․

สิก

का

के aे

$\doteq \dot{e}$

i̊

กิ

䨔

तิ สิ

ริ

00

00

กิ กิ

ตี

m

eे

ษ

e e

ษ. ․․ㅇ

กิ

e e

m.

กิ

i

ㅇ.

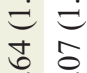

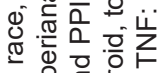

产高完产市

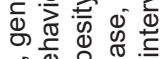

赵造造

흔 은

ปิ ปี ํำ

ปิ

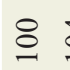

$\stackrel{m}{=}$

으

$\stackrel{n}{n}$ in

ต

莳

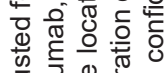

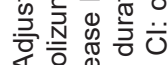

iิ

s

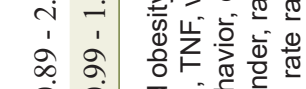

के

C.

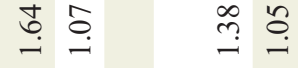

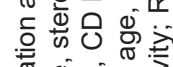

के

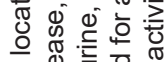

$\begin{array}{llll}0 & 0 & 0 & 0 \\ 1 & 1 & 1 & 1\end{array}$

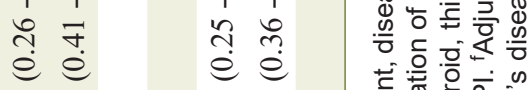

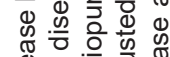

ज.

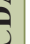

$-1$

\section{a}

$$
\text { n }
$$

$-$

$\sin$

0

하

$\vec{\sigma}$

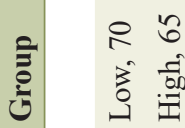

$\because$ in

高苟

㿣画

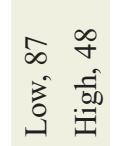

ก

ธ。

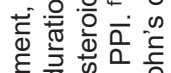

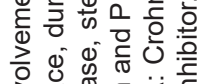

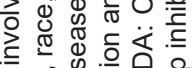

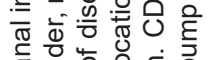

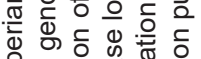

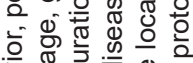

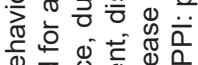

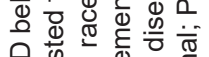

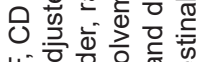


significant variation that might have occurred in their levels during observation. We also had to rely on cutoffs identified by Prometheus through their smart diagnostic algorithm and could not undertake our own independent validation. In future studies, the relationship between these serological markers and CD can be studied. Additionally, this study examined only clinical response and remission based on physician assessment and the HBI. Data on baseline radiologic, endoscopic or histological parameters were not collected nor were these additional parameters examined in conjunction with serological markers for predicting disease activity.

Nonetheless this study gives a real-world reflection of utility of serological markers in predicting disease activity in a tertiary care IBD referral center.

\section{Conclusion}

Serological markers have emerged as a noninvasive diagnostic test for IBD and can be employed in the diagnostic algorithm for IBD and differentiating UC from $\mathrm{CD}$. However, their role in predicting disease course is debatable and unclear. This is primarily due to lack of clinical trials comparing different serological markers and CD activity. There is a pressing need for large multicenter studies to assess the role of serological markers in predicting disease activity and their utility in deciding treatment options for complicated patients.

\section{Acknowledgments}

We would like to acknowledge all authors for their contribution towards the research paper.

\section{Financial Disclosure}

This study was not funded by any grant and the authors do not have any financial disclosures to make.

\section{Conflict of Interest}

The authors have declared no conflict of interest.

\section{Informed Consent}

The current study was approved by UAB's Office of Institutional Review Board and informed consent was waived.

\section{Author Contributions}

ZA contributed to study design, data collection and manuscript writing. ML contributed to data collection, and reviewed the manuscript for important intellectual content. NZ contributed to data analysis. TAM contributed to study design, and manuscript writing. TAM had full access to all of the data in the study and takes responsibility for the integrity of the data and the accuracy of the data analysis.

\section{Abbreviations}

ASCA: anti-Saccharomyces cerevisiae antibody; CD: Crohn's disease; EMR: electronic medical record; HBI: Harvey-Bradshaw index; IBD: inflammatory bowel disease; pANCA: perinuclear anti-neutrophilic cytoplasmic antibody; SGI: serum genetic inflammatory; UC: ulcerative colitis; VD: vedolizumab; UST: ustekinumab; MTX: methotrexate

\section{References}

1. Tomasello G, Scaglione M, Mazzola M, Gerges Geaga A, Jurjus A, Gagliardo C, Sinagra E, et al. Crohns disease and extra intestinal granulomatous lesions. J Biol Regul Homeost Agents. 2018;32(1):7-11.

2. Feuerstein JD, Cheifetz AS. Crohn disease: epidemiology, diagnosis, and management. Mayo Clin Proc. 2017;92(7):1088-1103.

3. Mitsuyama K, Niwa M, Takedatsu H, Yamasaki H, Kuwaki K, Yoshioka S, Yamauchi R, et al. Antibody markers in the diagnosis of inflammatory bowel disease. World J Gastroenterol. 2016;22(3):1304-1310.

4. Roggenbuck D, Reinhold D, Baumgart DC, Schierack P, Conrad K, Laass MW. Autoimmunity in Crohn's diseasea putative stratification factor of the clinical phenotype. Adv Clin Chem. 2016;77:77-101.

5. Quinton JF, Sendid B, Reumaux D, Duthilleul P, Cortot A, Grandbastien B, Charrier G, et al. Anti-Saccharomyces cerevisiae mannan antibodies combined with antineutrophil cytoplasmic autoantibodies in inflammatory bowel disease: prevalence and diagnostic role. Gut. 1998;42(6):788-791.

6. Yao F, Fan Y, Lv B, Ji C, Xu L. Diagnostic utility of serological biomarkers in patients with Crohn's disease: A case-control study. Medicine (Baltimore). 2018;97(32):e11772.

7. Means TK, Hayashi F, Smith KD, Aderem A, Luster AD. The Toll-like receptor 5 stimulus bacterial flagellin induces maturation and chemokine production in human dendritic cells. J Immunol. 2003;170(10):5165-5175.

8. Papadakis KA, Yang H, Ippoliti A, Mei L, Elson CO, Hershberg RM, Vasiliauskas EA, et al. Anti-flagellin (CBir1) phenotypic and genetic Crohn's disease associations. Inflamm Bowel Dis. 2007;13(5):524-530.

9. Targan SR, Landers CJ, Yang H, Lodes MJ, Cong Y, Papadakis KA, Vasiliauskas E, et al. Antibodies to CBir1 flagellin define a unique response that is associated independently with complicated Crohn's disease. Gastroenterology. 2005;128(7):2020-2028.

10. Coukos JA, Howard LA, Weinberg JM, Becker JM, Stucchi AF, Farraye FA. ASCA IgG and CBir antibodies are associated with the development of Crohn's disease and fistulae following ileal pouch-anal anastomosis. Dig Dis 
Sci. 2012;57(6): 1544-1553.

11. Gu P, Kapur A, Li D, Haritunians T, Vasiliauskas E, Shih DQ, Targan SR, et al. Serological, genetic and clinical associations with increased health-care resource utilization in inflammatory bowel disease. J Dig Dis. 2018;19(1):1523.

12. Reese GE, Constantinides VA, Simillis C, Darzi AW, Orchard TR, Fazio VW, Tekkis PP. Diagnostic precision of anti-Saccharomyces cerevisiae antibodies and perinuclear antineutrophil cytoplasmic antibodies in inflammatory bowel disease. Am J Gastroenterol. 2006;101(10):24102422.

13. Zholudev A, Zurakowski D, Young W, Leichtner A, Bousvaros A. Serologic testing with ANCA, ASCA, and
anti-OmpC in children and young adults with Crohn's disease and ulcerative colitis: diagnostic value and correlation with disease phenotype. Am J Gastroenterol. 2004;99(11):2235-2241.

14. Plevy S, Silverberg MS, Lockton S, Stockfisch T, Croner L, Stachelski J, Brown M, et al. Combined serological, genetic, and inflammatory markers differentiate non-IBD, Crohn's disease, and ulcerative colitis patients. Inflamm Bowel Dis. 2013;19(6):1139-1148.

15. Lichtenstein GR, Targan SR, Dubinsky MC, Rotter JI, Barken DM, Princen F, Carroll S, et al. Combination of genetic and quantitative serological immune markers are associated with complicated Crohn's disease behavior. Inflamm Bowel Dis. 2011;17(12):2488-2496. 